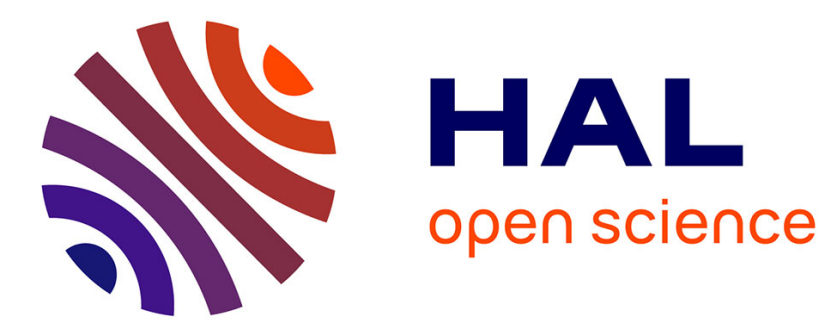

\title{
SEMI-CLASSICAL APPROACHES TO ELECTRIC GIANT RESONANCES
}

\author{
J. Treiner
}

\section{To cite this version:}

J. Treiner. SEMI-CLASSICAL APPROACHES TO ELECTRIC GIANT RESONANCES. International Symposium on Highly Excited States and Nuclear Structure Hesans 83, 1983, Orsay, France. pp.C4-265-C4-279, 10.1051/jphyscol:1984420 . jpa-00224086

\section{HAL Id: jpa-00224086 https://hal.science/jpa-00224086}

Submitted on 1 Jan 1984

HAL is a multi-disciplinary open access archive for the deposit and dissemination of scientific research documents, whether they are published or not. The documents may come from teaching and research institutions in France or abroad, or from public or private research centers.
L'archive ouverte pluridisciplinaire HAL, est destinée au dépôt et à la diffusion de documents scientifiques de niveau recherche, publiés ou non, émanant des établissements d'enseignement et de recherche français ou étrangers, des laboratoires publics ou privés. 


\title{
SEMI-CLASSICAL APPROACHES TO ELECTRIC GIANT RESONANCES
}

\author{
J. Treiner \\ Institut de Physique Nuclëaire, B.P. nº , 91406 Orsay Cedex, France
}

\begin{abstract}
Résumé - On présente une revue de diverses approximations conduisant à une description des Résonances Géantes en terme de dynamique des fluides, ainsi que des calculs semi classiques de règles de somme RPA. Le cas dipolaire, en particulier la polarisabilité dipolaire, est traité en détail, en relation avec les propriétés d'isospin de l'interaction. Une explication possible de la fragmentation de la fonction d'intensité RPA est donnée, qui la relie à des valeurs trop faibles du coefficient de symétrie de surface.
\end{abstract}

Abstract - Different fluid-dynamical approximations for Giant Resonances are reviewed. Semi classical calculations of RPA sum rules are presented. A detailed analysis of the Dipole case is made, in relation with the isospin properties of the effective interaction. The dipole polarizability is investigated. The unphysical fragmentation of the RPA dipole strength is discussed, and a possible explanation is given, which relates this erroneous feature to too low values of the surface symmetry coefficient.

\section{I - INTRODUCTION}

Electric Giant Resonances in nuclei are of collective character, so that one can expect shell effects to be of minor importance in their description; the smooth variation of the peak energies with mass number supports this idea. This fact calls for descriptions of the vibrations in terms of macroscopic variables (densities, displacements, currents ...) which are taken as basic variables of the theory in the different fluid dynamical and semi-classical approaches. Simplifying the dynamics, one can hope to gain more physical insight in the properties of the effective interaction involved in each type of resonance : the nuclear incompressibilities in the case of the Giant Monopole Resonance (GMR), the symmetry energy coefficients in the case of the Giant Dipole Resonance (GDR)... In the simple hydrodynamical picture one assumes that the static intrinsic hamiltonian remains valid in the dynamical case provided one replaces the static density by the time-dependent one. While this picture is valid for calculating for example static polarizabilities, it fails each time distorsions of the Fermi surface in momentum space generates restoring forces through changes in the kinetic energy. These forces (independent of forces which may arise from changes in the density) are of course not taken into account in the hydrodynamical mode1 which assumes a spherical Fermi surface(local equilibrium).

To overcome this difficulty, one has looked for approximations to the HF-RPA scheme allowing for these distorsions of the Fermi surface, based on the generalized scaling approximation $/ 1-4 /$ We shall briefly characterize in sect.II different methods along this line and some of the achievements and opened questions.

An alternative method can be used to characterize the RPA strength function $S(E)$ corresponding to a given excitation operator $Q$ : if the strength distribution is concentrated in a narrow region of energy (no fragmentation), the knowledge of a few moments of $S(E)$ suffices to extract the physical information. Indeed some of these moments can be calculated in a semi-classical framework as will be described in sect. III, and this method has been extensively used in the description of the GMR/5-8/, and to a lower extent to the GDR/9-13/.The extension of the method to $\mathrm{fi-}$ nite temperatures is straightforward and will give informations on Giant Reso- 
nances built on excited states, for which experimental data become available (see Snover's talk to this conference) : displacement of peak energies and evolution of escape widths with excitation. In the present work (sect. IV), we shall discuss in some details the Dipole case in relation with the symmetry eneray coefficients: besides the Dipole polarizability, we shall discuss the (unphysical) fragmentation of the strength obtained in RPA calculations with commonily used interactions, even when giving satisfactory results concerning the moments ; we shall see that the transparency of the semi classical approach to the RPA sum rules allows one to give argument that this wrong feature is related to erroneous values of the surface symmetry coefficient.

\section{II - SOME REMARKS ON THE FLUID DYNAMICAL APPROACH}

Detailed discussions of the generalyzed scaling approximation can be found in refs. $\mid 14-16 /$. Here we just want to sketch the main points which will be useful when discussing the Dipole case in the next section.

When boking for approximations to the HF-RPA, one is guided by the structure of the solution when only one state exhausts the sum rule. In that case, it was shown by Bertsch $/ 1 /$ that the solution can be obtained by applying TDHF to a scaled ground state $\psi$ which, in its most general form is written $/ 2,4 /$

$$
\left|\psi>=e^{i m \sum_{i} \xi\left(\vec{r}_{i}, t\right)} e^{\alpha(t) \sum_{i} \overrightarrow{\nabla S}\left(\vec{r}_{i}, t\right)+\vec{s}\left(\vec{r}_{i}, t\right) \cdot \vec{\nabla}}\right| 0>
$$

The scalar field $\xi$ generates the current and one can show that the resulting flow is irrotational, the velocity being given by

$$
\vec{v}=-\dot{\vec{s}}=\vec{\nabla} \xi(\vec{r}, t)
$$

The collective kinetic energy has just the classical form

$$
B(\vec{s})=\frac{1}{2} m \int \rho(\vec{r})(\vec{\nabla} \xi)^{2} d \vec{r}
$$

in agreement with the general results of ref. $117 /$, and the collective potential energy $C(\vec{s})$ is a functional of the scaling field $\vec{s}$. The frequency of the mode can be written as

$$
\omega^{2}=C(\vec{s}) / B(\vec{s})
$$

Afluid dynamical scheme is obtained by considering the scaling ansatz the general case and applying the variational principle

$$
\delta \int_{t_{1}}^{t_{2}}\left\langle\psi\left|H-i h \frac{\delta}{\delta_{t}}\right| \psi\right\rangle=0
$$

in order to derive the equations of motion. At this point, it should be stressed that variations can be taken ejther with respect to $\xi$ or with respect to $\vec{\nabla} \xi$, as $\xi$ appears in eq. (5) onty through its gradient. In the first case, one is led to an irrotational fiuid dynamical picture $/ 16 /$ whereas in the second case the solutions are in general rotational/4,14/.

In this last case, Holzwarth et al., assume that the classical expression for the mass parameter is still valid, which might be questionable in some cases. We shall see in particular that in the dipole case, the fragmentation of the strength predicted by the rotational fluid dynamical approach does not correspond to the 
microscopic RPA one. There are good indications/18/ that just one scalar field $\xi(\vec{r}, t)$ is not enough to describe the fragmentation of the strength, especially when a low-lying state exists (quadrupole or octupole case) and the same is probably true in the dipole case also where the fragmentation is not linked to shel1 effects. Including non local operators in the time odd part of the scaling operator in order to generate rotational components is being worked out by da Providencia and Hol.zwarth/19/.

A comparison between irrotational fluid dynamics and RPA can be found in ref./16/ for the isoscalar case (monopole and quadrupole). The agreement is found excellent, for the strengths as well as for the currents, in the case where the RPA strength is not fragmented. In the isovector case, the comparison is also very satisfactory in the same conditions (see Ando and Eckart's contribution to this conference), namely i) no low-lying state in the RPA strength $i j)$ the resonance peak is well separated from the energy region where the unperturbed strength $1 i$ es and $i i_{i}$ ) the HF ground state is used as input in the dynamical calculation (instead of a semi classical one).

Let us conclude these few remarks by noticing that the structure of the eigen modes can be simply analyzed in the framework of the generalized scaling. Studies in this sense can be found in refs./20,21/. Recent]y/22,23/,eq.(4) has been used in the study of the coupling between surface and bulk vibrations in the isoscalar monopole resonance. The distorsion of the Fermi surface is neglected here, which is justified in the scaling model of the GMR. Parametrizing the transition density $\delta \rho$ in a simple form exhibiting the desired feature to be discussed, the velocity field is obtained by integrating the continuity equation. Minimizing $\omega$ in eq. (4) with respect to the parameters characterizing sp (using a semi classical approximation for the calculation of C) gives the eigen-modes of the system. While the lower state (identified to the experimental GMR) is found to be close to the scaling model (stretching of the nuclear surface in phase with the compression of the interior) another state at higher energy shows an "antiscaling behaviour". This result is in qualitative agreement with the more detailed study of ref./21/.

The corresponding excitation operator can be obtained by integrating the velocity field (at least if one assumes that the transverse component is negligible); it would then be interesting to investigate, using the same operator, whether RPA calculations confirm the semi classical results, in view of the fact that possible distorsions of the Fermi surface, negligible for the first excited state, may not be negligible for the second monopole state.

III - SEMI CLASSICAL CALCULATIONS OF RPA SUM RULES

As mentioned in the introduction, if the resonance is highly collective, one can hope that the knowledge of a few moments $m_{k}$ will give the salient features of $S(E) / 24 /$

$$
\begin{aligned}
& S(E)=\sum_{n} \mid\langle n|Q| 0\rangle^{2} \delta\left(E-E_{n}\right) \\
& m_{k}=\int E_{n}^{k} S(E) d E
\end{aligned}
$$

In a semi classical framework, where one uses some extended Thomas Fermi approximation for the kinetic energy, i.e. one assumes a spherical Fermi surface, the $m_{1}$ and $m_{1}$ moments can be easily evaluated. The polarizability $p=2 m_{-1}$ can be obtained as the response of the system to a constraining external field $\hat{Q}$, i.e. one solves the static Euler equations corresponding to $H-\lambda$. Then one has

$$
m_{-1} \equiv \sum_{n} \frac{1}{E_{n}}|\langle n|Q| 0\rangle|^{2}=\frac{1}{2} \frac{d^{2} E(\lambda)}{d \lambda^{2}}
$$

The $m_{1}$-moment is related to a simple ground state property. For example in the monopole case $\left(Q=r^{2}\right)$, one has

$$
m_{1}=\frac{2 \hbar^{2}}{m} A\left\langle r^{2}\right\rangle
$$


and in the dipole case :

$$
m_{1}=\frac{\hbar^{2}}{m} \frac{N Z}{A}(1+k)
$$

where $\kappa$ is the so-called enhancement factor, related to the exchange component of the interaction ( $\equiv$ velocity dependent part of the interaction in case of a Skyrme interaction).

In general, the calculation of the $m_{3}$-moment involves deformations of the Fermi surface, as it is obtained through a scaling of the quantum ground state/24/. However it happens that in the monopole and in the dipole case, these deformations do not occur, so that a semi classical calculation using the static functional for the kinetic energy is possible.

In the isovector case, one has to treat correctly the exchange part of the interaction, which modifies the classical expression for the collective kinetic energy (see ref./9/).

Knowing $m_{-1}, m_{1}$ and $m_{3}$, one can have an estimate of the escape width by considering,

$$
\sigma^{2}=\frac{1}{4}\left(\frac{m_{3}}{m_{1}}-\frac{m_{1}}{m-1}\right)
$$

The method described above has been used in the past few years in the description of the GMR, in particular concerning the choice of the sum-rules appropriate in extrapolating nuclear incompressibilities from finite nuclei to the nuclear matter value

$$
K_{n m}=9 \rho_{n m} \quad \frac{d^{2} E / A}{d \rho_{n m}^{2}}
$$

Without entering into details we shali briefly recall here the main results obtained in ref./8/. Defining the incompressibility $\mathrm{K}_{A}$ of a nucleus from the position of the resonance energy $E_{M}$ by

$$
E_{M}=\sqrt{\frac{m_{3}}{m_{I}}}=\sqrt{\frac{\hbar^{2}}{m} \frac{K A}{\left\langle r^{2}\right\rangle}}
$$

we can write an $A^{-1 / 3}$-expansion of $K_{A}$ :

$$
K_{A}=K_{V}+K_{S} A^{-1 / 3}+K_{\delta}\left(\frac{N-Z}{A}\right)^{2}+K_{\text {cout }} \cdot \frac{Z^{2}}{A^{4 / 3}}
$$

A fit to experimental data does not allow to extract the values of $K_{y}, K_{S}$, $K_{\delta}$ and

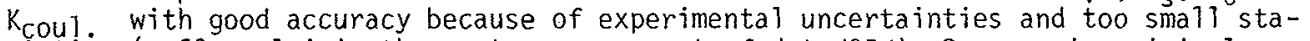
tistics ( $~ 60$ nuclei in the most numerous set of data/25/). Some semi-empirical relations between the coefficients can be obtained by considering the calculated incompressibilities. One can show that expansion (12) is rapidly converging (this would not be the case when considering $\sqrt{\frac{m_{1}}{m_{1}}}$, and that $k_{z_{i}}=k_{n m_{b y}}$. The coulomb
term can be well approximated, for a large variety of interactions

$$
K_{\text {cour }}=-\frac{3}{5} \frac{e^{2}}{r_{0}}\left(12.5-\frac{1215}{K_{n m}}\right)
$$

where $r_{0} \cong 1.15 \mathrm{fm}$ and $K_{n m}$ is in MeV. Detailed investigations also show that

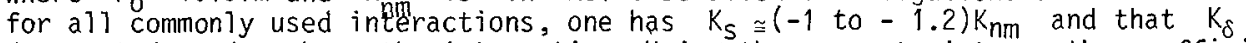
does not depend much on the interaction. Using these constraints on the coefficients, one can use the different sets of experimental data to determine by a fit the value 
of $K_{n m}$ only. The following results are obtained

$$
\begin{aligned}
& K_{n m}=220 \pm 30 \mathrm{MeV} \\
& K_{s}=-240 \pm 70 \mathrm{MeV} \\
& K_{\delta}=-300 \pm 150 \mathrm{MeV}
\end{aligned}
$$

We show in Fig. 1 the evolution of $K_{A}$, as given by eqs. 13 and 14 , as a function of mass number. Experimental points are given for comparison. In medium nuclei, they lie below the semi-theoretical curve. However one should keep in mind that the percentage of EWSR exhausted by the experimental strength decreases from $100 \%$ in heavy nuclei down to a few percent in light systems. The experimental value of $K_{A}$ deduced from the peak energy, even when taking into account the experimental width in order to calculate $\sqrt{\frac{m_{3}}{m_{1}}}$ probably underestimates the true value if some strength is missing at higher energy.

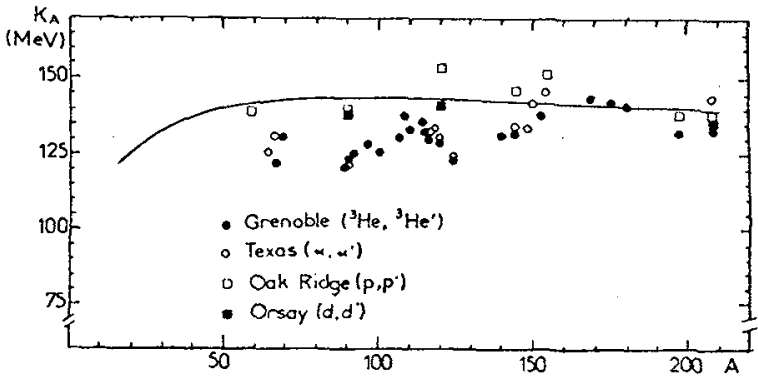

Fig.1 - Values of empirical incompressibilities. The line corresponds to incompressibilities obtained using eq.(12) with values of the coefficients given in eqs. (13) and (14).

\section{IV - THE GIANT DIPOLE RESONANCE AND THE SYMMETRY ENERGIES}

We sha 11 now discuss the isospin properties of the effective interaction - in particular the volume and surface symmetry coefficients $J$ and $\varepsilon_{s s}$ - in relation to the GDR.

These quantities are known to play a role in various situations : ground state masses and especially nuclei far from the stability ine, which are of astrophysical interest, neutron skin and fission barrier heights. However a clear determination of both coefficients separately is not easy; the values of $J$ lie in the range $(30-37) \mathrm{MeV}$ while the values of $\varepsilon_{s s}$ found in the literature vary from $-30 \mathrm{MeV}$ to $-160 \mathrm{MeV}$ in the Droplet Model /26/ Falthough in this latter case, strictly speaking, $\varepsilon_{S S}$ is not well defined). It is then interesting to investigate wether the GDR can bring some constraints on these two coefficients $/ 27 \%$. We sha11 be concerned, in the following, with the static dipole polarizability $p$, related to the moment $m_{2}$ and to the integrated cross section by

$$
p=2 m_{-2}=4 \pi^{2} \frac{\hbar c}{e^{2}} \sigma_{-2}=4 \pi^{2} \frac{\hbar c}{e^{2}} \int \frac{\sigma(\omega)}{\omega^{2}} d \omega
$$

The $m_{1}$ moment is given by eq.(9) and we calculate $m_{3}$ using a dipole scaling . 


\section{IV.1 - Surface effects on the dipole_polarizability}

The dipole polarizability $p$ is a rather well known quantity over the mass table. Due to the weighting factor $\omega^{-2}$ in eq. (15), it is not sensitive to the high energy part of the cross-section, i.e. to short range correlations. Hence it is a good quantity to which effective interaction used in H.F. and RPA calculations should be tested.

The role of the diffuse nuclear surface on the dipole polarizability $p$ can be identified by looking at the general trend of the quantity $\sigma_{-2} A^{-5 / 3}$ over the mass table. A constant density model leads to Migdal's formula involving the volume symmetry energy $J$ :

$$
\sigma_{-2}^{M}=2 \pi^{2} \frac{e^{2}}{\hbar c} A \frac{\left\langle r^{2}\right\rangle}{24 J} \cong 2 \pi^{2} \quad \frac{e^{2}}{\hbar c} \quad \frac{r_{0}^{2}}{40 J} A^{5 / 3}
$$

with $r_{0} \cong 1.15 \mathrm{fm}$. Fig.2 shows the experimental points, plotted versus $A^{-5 / 3}$ in order to visualize more clearly finite size effects. The error bar in the nuclear matter value corresponding to Migdal's formula is obtained by considering two extreme values of $J$, namely $\mathrm{J}=28 \mathrm{MeV}$ and $\mathrm{J}=37 \mathrm{MeV}$ (this last value is favoured by recent mass formula fits). One sees that surface effects are indeed important : the experimental value in the region of medium and heavy nuclei, aimost constant $\cong(2.9 \pm 0.2) \mu b \times \mathrm{MeV}^{-1}(1)$, is $250 \%$ greater than the largest estimate of the Migdal value $\sigma_{-2}^{M}$ obtained using a low value of $\mathrm{J}$. For example taking $\mathrm{J}=28 \mathrm{MeV}$, one gets $\sigma_{-2}^{M} A^{-5 / 3} \cong 1.85 \mu \mathrm{bMeV}^{-1}$. In 1ight systems, the experimental value of $\sigma_{-2} A^{-5 / 3}$ is larger by a factor 2 or 3 .

This trend has been confirmed by RPA calculations/29/, and semi classical /10/ and hydrodynamical/11,13/investigations have analyzed in more details the dependence of $\sigma-2$ on the isospin properties of the effective interaction.

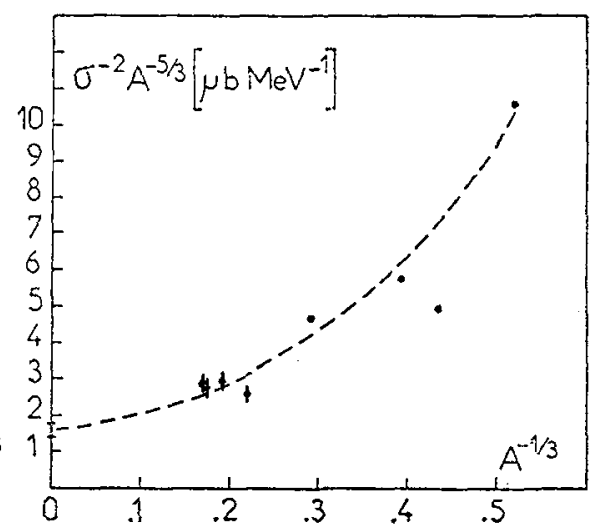

The energy density formalism used in ref $/ 10 /$ gives results in remarkable agreement with the RPA calculations of ref. /29/. The polarizability is obtained through a constrained calculation, i.e. one minimizes the energy of the system under the constraint of a dipole operator $\mathrm{D}_{z}$. The transition density is obtained as the solution of a differential equation and the calculation is fully self-consistent. Fig. 3 shows the result in case of calcium and lead, One has a volume type of deformation, corresponding to the Steinwedel Jensen model, but of course with a diffuse surface. In order to investigate the role of $J$ and $\varepsilon_{S S}$, one can change separately both
quantities, by changing the symmetry potential as a function of the density (see ref. $/ 36 /$. Fig.(4) shows the results of 4 sets of calculations corresponding to varying the values of $\varepsilon_{\text {ss }}$ while keeping $J$ fixed. Surprisingly, it is seen

(1) This value includes, besides the neutron emission cross section, the inelastic photon scattering cross section below neutron threshold. Such measurements have been made in nuclei near ${ }^{208} \mathrm{~Pb} / 28 /$, giving a contribution of $\sim 7 \%$ to $\sigma_{-2}$. We have adopted this value in the mass region $A>100$. 
that very different couples of values of $J$ and $\varepsilon_{S S}$ lead to similar agreement with experiment.

(The experimental increase of $\sigma_{-2} A^{-5 / 3}$ when going from heavy to light nucle $i^{-2}$ tends to be larger than the theoretical one : part of the disagreement may be due to the neglect of the spin orbit interaction when solving the constrained problem; the spin orbit contribution to the residual interaction is also neglected in RPA calculations; another source of disagreement may lie in the inclusion of the $(\gamma, p)$ channel which has not been measured in the mass region $A>100$ or by the process of subtracting the nonnuclear part of the total photo absorption cross section in light nuclei).
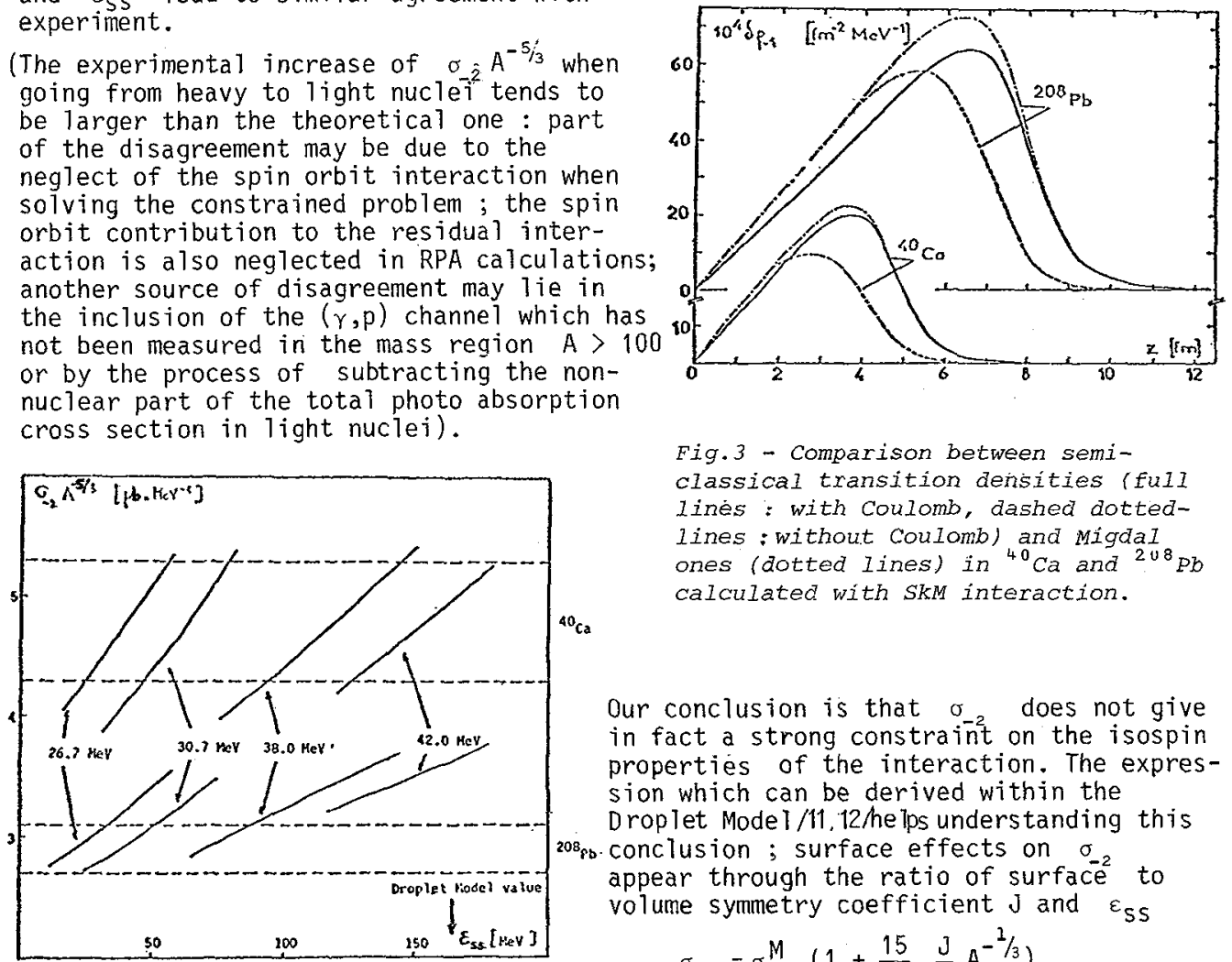

Fig.3 - Comparison between semiclassical transition densities (fuII lines : with Coulomb, dashed dottedlines : without Coulomb) and Migdal ones (dotted lines) in ${ }^{40} \mathrm{Ca}$ and ${ }^{248} \mathrm{~Pb}$ calculated with SkM interaction.

Our conclusion is that $\sigma_{-2}$ does not give in fact a strong constraint on the isospin properties of the interaction. The expression which can be derived within the D roplet Model $/ 11,12$ helps understanding this ${ }^{208_{\mathrm{pb}} \text {-conclusion; surface effects on }}$ appear through the ratio of surface to volume symmetry coefficient $J$ and $\varepsilon_{\text {SS }}$

$$
\begin{aligned}
\sigma_{-2} & =\sigma_{-2}^{M}\left(1+\frac{15}{4} \frac{J}{Q} A^{-1 / 3}\right) \\
& =\sigma_{-2}^{M}\left(1+\frac{5}{3} \frac{\varepsilon_{S S} A^{-1 / 3}}{J}\right)
\end{aligned}
$$

surface symmetry coefficient $\varepsilon_{\text {ss }}$ for different values of the volume symmetry energy $J$.

where $Q$ is the so-called stiffness parameter. However this relation, while describing correctly the qualitative behaviour of $\sigma$, should not be used as such to analyze experimental data : besides Coulomb effects which are neglected in eq. (17) and which lower the values of $\sigma_{-2}$ by $210 \%, \mathrm{Fig.2}$ shows that $A^{-2} \sqrt{3}$ corrections are indeed not negligible if one wants to analyze $\sigma_{-2}$ on the whole mass table. On the other hand, if one restricts oneself to heavy systems where one expects curvature effects to be small eq.17 gives only a correlation between $J$ and $\frac{\varepsilon_{S S}}{J}$ : similar values of $\sigma$ are obtained with low or high values of both $\mathrm{J}$ and $\frac{\varepsilon_{S S}}{\mathrm{~J}}$,

Other moments of the strength distribution

Besides $m_{2}$, the moments $m_{7}$ and $m_{3}$ can be calculated in an energy density formalism, and used to characterize the energy of the resonance by considering the ratios

$$
E_{2}=\sqrt{\frac{m_{1}}{m_{-1}}} \text { or } E_{3}=\sqrt{\frac{m_{3}}{m_{1}}}
$$

(there is no theoretical expression for the peak energy). 
The $m_{1}$ moment is related to the dipole enhancement factor $k$ $m_{1}=60 \frac{N Z}{A}(1+k) m b . M e V$

Considering $E_{1}$ does not bring any new physical information on the isospin properties of the interaction because $m_{1}$ does not depend on these properties. This is not the case with $E_{3}$, as $m_{3}$, which can be obtained through a dipole scaling on the ground state (taking into account the exchange terms of the interaction ), corresponds in fact to the Goldhaber-Teller model, i.e. to a vibration in the surface-hence sensitive to surface properties- as can be seen from the approximate expression obtained in ref./9/:

$$
E_{3}=\left[\frac{2}{3} \cdot \frac{\hbar^{2}}{m} \cdot \frac{\int\left[\rho^{\prime}(1+2 \times \rho)\right]^{2} \cdot \rho \cdot \varepsilon_{\delta}(\rho) d \vec{r}}{A(1+K)}\right]^{1 / 2}
$$

where $k=x \rho_{0}$ ( $\rho_{0}$ is the central density) and $\varepsilon_{\delta}(\rho)$ represents the symmetry potential as a function of density $\left(\varepsilon_{\delta}\left(\rho_{0}\right)=J\right)$.

Notice the weighting factor $\rho^{12}$ in the numerator showing that $E_{3}$ is indeed sensitive to the values of $\varepsilon_{\delta}(\rho)$ at surface ( $(\equiv$ small) densities, i.e. to the surface symmetry coefficient (see below). Identifying $E_{3}$ with the peak energy has led to values of $\varepsilon_{S S} \sim 2$, as for the SkM interaction $/ 9 /$ which, after slight modification has allowed to get - for the first time - good agreement with experiment for the fission barrier height of $240 \mathrm{Pu} / 30 \%$

Eq. (18) shows that $m_{3}$ is proportional to $(1+k)^{2}$, so that $E_{3}$ is proportional to $\sqrt{1+k}$. This raises a problem when comparing with experiment. The experimental value of $k$, obtained by integrating the photoabsorption cross section up to $140 \mathrm{MeV}$ are discussed in details in ref. $131 \%$. In medium and heavy nuclei one finds an almost constant value $k=0.76 \pm 0.10$. However effective interactions used in HF-RPA calculations should not reproduce such a high value, because the method is not able to describe the physics above a few tens of MeV (short range and tensor correlations become important). Therefore the values of $k$ commonily used are smaller and 1 ie in the range $0.35-0.45$ al though one does not have an objective criterium to determine this value.

In order to get rid of this arbitrariness, the authors of ref./11/ propose to consider the ratio $\omega_{D}=\sqrt{\frac{m 3}{1(1+k)}}$ which does not depend on $k$. The corresponding experimental values of $\omega_{D}$ are shown not to depend much on the maximum energy used in the calculation of the moments and $\omega_{D}$ is found to be close to the peak energy. The conclusion drawn in ref./11/ is also $\frac{\varepsilon_{S S}}{J} \sim 2$, as above.

We shall now turn to microscopic calculations as, surprisingly, the RPA crosssections obtained with interactions giving satisfactory results concerning the moments of the strength function have always shown an unphysical feature which has to be understood.

\section{IV.2 - RPA_calculations_of the dipole_strength}

Fig.5 shows a typical feature of al] RPA calculations made so far with various kind of effective interactions /32\%. The present calculation is made using SkM interaction. This force gives satisfactory results concerning the moments; however the photoabsorption cross section in lead shows a fragmentation which is not seen experimentally : the strength shows main ly two concentrations in energy, separated by 4 to $5 \mathrm{MeV}$; most of the strength is concentrated in the lower part, which corresponds more or less to the experimental peak. 
The presence of the unphysical upper peak raises the following alternative : either its origin lies in some particularity of the particle-hole spectrum and it will disappear when more complex configurations $(2 \mathrm{p}-2 \mathrm{~h})$ are included in the calculation or ii) the fragmentation of the strength is linked to a macroscopic aspect and indeed reveals some eironeous macroscopic property of the effective interaction which will survive even after including some damping mechanism. In the first case, one should not be preoccupied by the presence of some structure in the strength : coupling to $2 p-2 h$ configurations might distribute the strength concentrated in the upper peak over a few MeV, giving some skewness to the shape of the resonance peak just as the experimental one shows. However calculations of the damping, although becoming available, /33/ are still too preliminary to answer the quantitative question of how much fragmentation can be accepted at the RPA lever. Besides, fluid dynamical

Fig. 5 - RPA photo absorption cross-section calculated in ${ }^{208} \mathrm{~Pb}$ with SkM interaction. Dashed line: experimental curve. calculations also give a fragmentation of the dipole strength /34/, which can be interpreted as resulting from the coupling of transverse to longitudinal components in the solutions of the equation of motion $/ 15 /$. It is then interesting to explore the second branch of the alternative mentioned above.

A first hint concerning the macroscopic aspect of the discussion is given by considering the transition densities in the two energy regions (they will be shown below), or more simply to the averaged transition densities $\delta_{p \pm 1}$ defined by:

$$
\delta_{\rho_{ \pm 1}}=\frac{1}{m \pm 1} \sum_{n}^{\sum} E_{n}^{ \pm 1}\langle 0|\vec{p}| n\rangle\left\langle n\left|D_{z}\right| 0\right\rangle
$$

where $\hat{\rho}$ is the density operator. The curves in Fig. 6 are calculated in ${ }^{208} \mathrm{~Pb}$ with SIII interaction $/ 35 \%$. Obviously $\delta \rho_{+1}$ favors states in the upper peak whereas $\delta \rho_{-1}$ favors low energy states. Now one can show that $\delta \rho_{+1}$ corresponds to a dipole scaling on the ground state density (GT mode 1 ), while $\delta p_{-}$is the change in the density when constraining the nucleus with a dipole operator (in other words $\delta \rho_{-1}$ in Fig. 5 is just the quantum analog to Fig. 2). This indicates, as already noticed by Bertsch and Tsai in ref./32/ that the upper states are of surface type (although as we shall see below by looking at the currents, they do not correspond to the GT. model because of the important transverse components) while the lower states are more of SJ type. Consequently the upper states should be more sensitive to the surface symmetry energy.

Another hint, confirming the first one, can be found in eq. (18) for $E_{3}$. As a lready mentioned, due to the $\rho^{12^{3}}$ weighting factor in the numerator of eq. (18), $E_{3}$ is

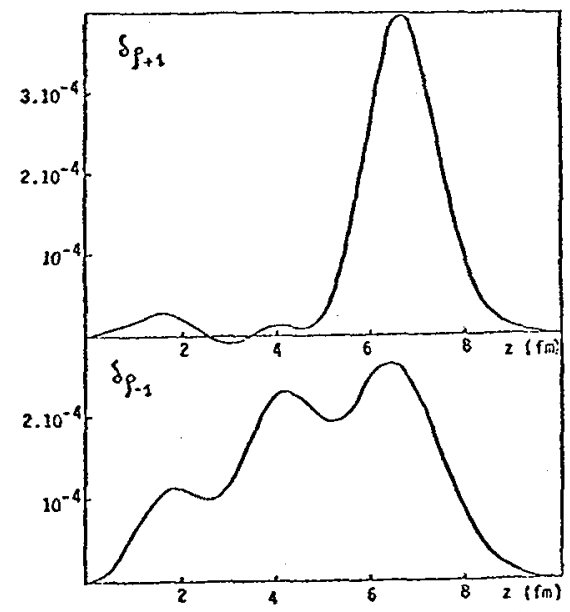

Fig.6-Averaged transition densities $\delta p_{ \pm 1}$ (eq. (19)) calculated in ${ }^{208} \mathrm{~Pb}$ with $s$-III interaction. 
sensitive to the behaviour of the symmetry potential $\varepsilon_{\delta}(\rho)$ at surface densities. Smaller values of $\varepsilon_{\delta}(\rho)$ at half nuclear density should decrease the value of $E_{3}$ i.e. should tend to make the unphysical peak disappear. Now decreasing $\varepsilon_{\delta}(\rho)$ at low densities corresponds to increasing the surface symmetry energy. Indeed by considering the semi-infinite medium, one can derive, within an energy density formalism, the following approximate expression for $\varepsilon_{\mathrm{SS}} / 36 /$

$$
\varepsilon_{S S}=8 \pi r_{O}^{2} \quad \int_{-\infty}^{+\infty} \rho\left(\varepsilon_{\delta}(\rho)-J\right) d x
$$

which shows that $\varepsilon_{\text {SS }}$ can be interpreted as the defect of symmetry energy (with respect to the uniform value $J$ at saturation), integrated over the surface. This defect is of course the more negative as $\varepsilon_{\delta}(\rho)$ is small a low densities. Hence increasing (in absolute value) Ess should make the upper peak disappear, either by moving its value downward, or by transferring the strength to the lower peak.

In order to check this hypothesis, we have made different sets of RPA calculations in the following model conditions (an averaging energy interval of $1 \mathrm{MeV}$ is used)

* no velocity dependent Skyrme type interaction : no approximations are then made in the RPA calculation

* $N=Z=70$ nucleus (no Coulomb, no spin-orbit) = the unperturbed cross-section plotted in Fig. 7 is thus independent of the isospin properties of the interaction.

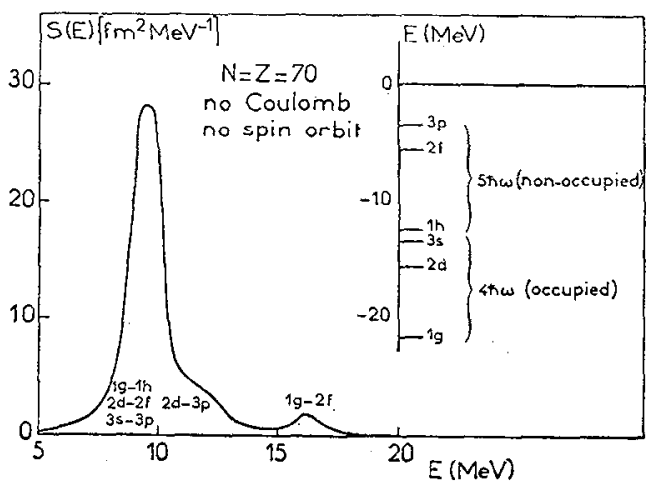

Fig. 7 - Unperturbed strength function used in the model calculation of $\mathrm{Fig.8}$.

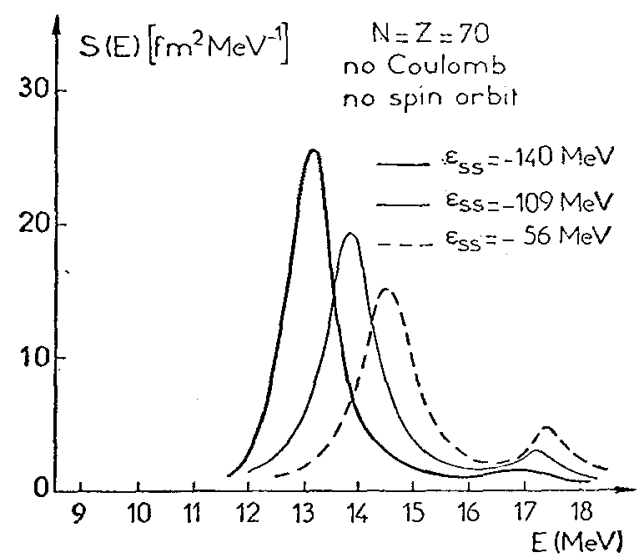

Fig. 8 - Effect of the surface symmetry coefficient. $\varepsilon_{s s}$ on the fragmentation of the strength.

Fig. 8 shows the results obtained for the RPA strength function when, fixing the volume symmetry $\mathrm{J}=37 \mathrm{MeV}$, one changes the surface symmetry energy $\varepsilon_{S S}$ (one varies the couple of exchange coefficients $x_{0}$ and $x_{3}$ of the Skyrme force). One sees that, in agreement with the qualitative conclusions of the semi classical approach, one has to go to high values of $\varepsilon_{S S}$ in order to make the unphysical peak disappear. Notice that the energy of the higher peak does not change much ; it is rather the strength which is transferred to the lower peak. The energy of the lower peak is shifted down by $2 \mathrm{MeV}$, but in a realistic case, if one chooses a large value of $\varepsilon_{s s}$ one should also increase $J$ because the mass of a nucleus with a neutron excess should remain constant. Then the shift of the lower peak is not so large. This can be seen on Fig. 9 , where are plotted the RPA-strength functions for ${ }^{208} \mathrm{~Pb}$ 
calculated with two different effective interactions giving the same binding energy (Coulomb and spin orbit forces are now included). The first one is the original SkM force, the second one has exactly the same isoscalar properties but $J$ has been increased to $37 \mathrm{MeV}$ and $\varepsilon_{S S}$ to $-140 \mathrm{MeV}$ (these values are very close to the Droplet Mode 1 constants). The result is the same as in the model case ; the unphysical peak is no longer present in this second case, i.e. for a ratio $\left|\frac{\varepsilon_{S S}}{\mathrm{~J}}\right| \sim 4$. Only a very weak structure is seen at $\sim 15 \mathrm{MeV}$ whereas in the case of SkM, the upper peak at $\sim 16 \mathrm{MeV}$ exhausts $\sim 30 \%$ of the EWSR.

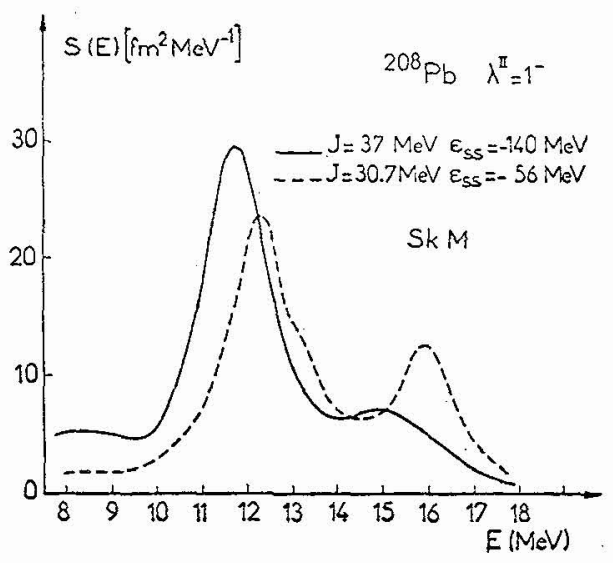

Fig. 9 - RPA strength functions in ${ }^{20 B} \mathrm{Bb}$ calculated with $S k M$ and with a modified force having $J=37 \mathrm{MeV}$ and $\varepsilon_{S S}=-140 \mathrm{MeV}$.
Now that we have shown that the fragmentation of the RPA strength is related to the isospin properties of the interaction, we want to stress that our aim is not to give a definite value for the ratio $\frac{\varepsilon_{s s}}{j}$. As long as one does not know how much structure can be washed out by coupling to $2 p-2 h$ configurations, one cannot get a precise answer, although there is probabiy too much fragmentation with the interactions commoniy used up to now. Besides, the position of the peak for the modified SKM interaction in Fig.(g) is too low compared to experiment, so that $m_{1}$ is too large. One could then move the position of the peak upward by taking a lower value of the effective mass, which would shift the unperturbed strength upward. Other physical phenomena where isospin properties are involved have also to be taken into account simultaneously: the neutron skin increases with increasing surface symmetry coefficient, the proton r.m.s. radius in ${ }^{48} \mathrm{Ca}$ decreases and the fission barrier height decreases. Such a study lies beyond the scope of the present work, where we just want to show a possible mechanism by which the unphysical feature of the RPA strength can be corrected.

We now turn to the study of the transition densities and convection currents. Fig. 10 shows the transition densities $\delta \rho(r, E)$ as functions of $r$ for different

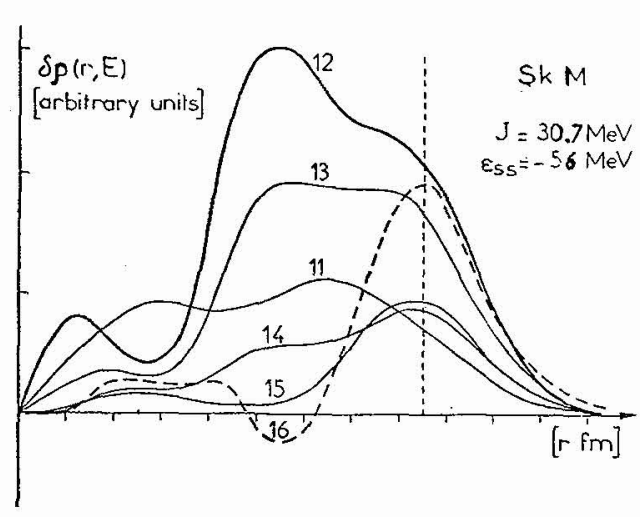

Fig. 10 - Plot of the transition densities at different energies calculated with SkM interaction.

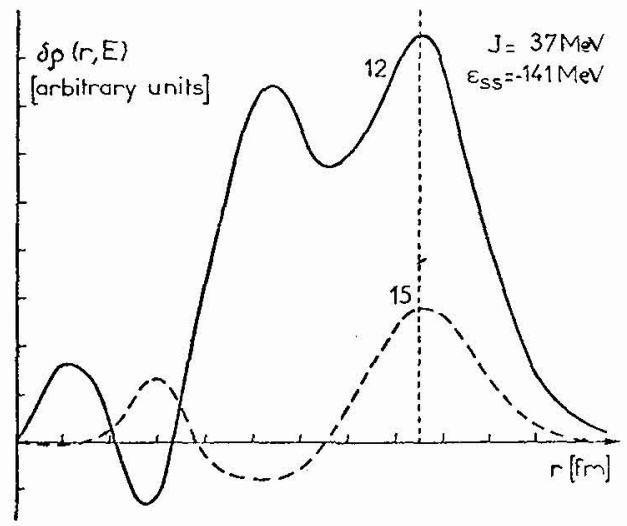

Fig. 11 - Same as Fig. 10 using the modified force referred to in Fig. 9. 
energies between 11 and $16 \mathrm{MeV}$ for the SkM-force. As expected, the amplitude of the vibration is maximum near $E=12 \mathrm{MeV}$ and $E=16 \mathrm{MeV}$, i.e. at peak energies. The dashed vertical line locates the nuclear surface; one sees that the lower peak is of volume type while the upper one is a surface vibration.

It is interesting to notice the change in $\delta \rho(r, E)$ around $12 \mathrm{MeV}$ when one increases the surface symmetry coefficient (in absolute value) to $140 \mathrm{MeV}$.

A large bump appears now at the surface (see Fig. 11), while the amplitude of $\delta \rho(r, E)$ around $E=15 \mathrm{MeV}$ has been significantly reduced. (The intermediate energies are not considered for clarity in Fig. 11). Indeed, large values of $\varepsilon_{S S}$ means that one does not loose much energy when separating neutrons and protons at the surface. The resonance peak appears now as a mixture of volume and surface vibration.

We have checked this behaviour in the model calculations also : there is always a clear connection between the values of $\varepsilon_{S S}$ and the shape of the transition density corresponding to the resonance, so that if one would be able to determine $\delta \rho$ experimentally, one would have another source of information on $\varepsilon_{S S}$.

The corresponding current in ${ }^{208} \mathrm{~Pb}$ are given in Fig. 12, in the case of the force with the large surface symmetry coefficient. In fact the qualitative features of the figs. do not depend much on the interaction. The vertical axis is the z-axis and one represents one fourth of the nucleus. At low energy the flow lines tend to be parallel to the surface except for large $z$ where the large value of $\boldsymbol{\varepsilon}_{5 S}$ allows for a crossing of the surface consistent with the above remark about the bump in the transition density. When going to higher energies, the flow lines tend to become parallel to the z-axis, i.e. to be more of Goldhaber-Teller type, but surprisingly this happens at the minimum of the strength function. Around 15 to $16 \mathrm{MeV}$ a vortex appears for small values of $z$ in the surface region: this was indeed unexpected as one would have rather predicted in this region a GT mode which is irrotational, whereas one has in fact large transverse components in the vortex.

Finally it is interesting to compare the type of fragmentation that we have obtained in the RPA calculations to the results of the fluid dynamical approach of ref. $134 /$. We notice a clear disagreement concerning both the strengths and the currents : i) we obtain more strength in the lower peak than in the higher one - which can eventually even disappear - while it is the contrary in ref./34/ ii) in RPA the low peak is to be identified with the Giant Resonance, so that the velocity field has small transverse components, and a vortex is seen at higher energies; in ref./34/ it is the low energy state which shows vorticity and the second state is almost irrotational. As aiready mentioned the disagreement might be linked to the treatment of the collective kinetic energy in the calculation of the transverse component.

In conclusion, we want to stress the following points.

1) Semiclassical calculations of RPA moments are reliable and provide a useful tool in exploring the properties of nuclear effective interactions used in microscopic calculations.

2) In the case of the GDR, agreement with experiment is obtained for the polarizability for different couples of values of volume and surface symmetry coefficients. The unphysical fragmentation of the calculated RPA strength in ${ }^{208} \mathrm{~Pb}$ can be accounted for by too low values of the surface symmetry energy. The present analysis favors values of $\mathrm{J} \cong 35$ to $37 \mathrm{MeV}$ and of $\varepsilon_{\text {SS }}$ around $-140 \mathrm{MeV}$. However one should keep in mind that the damping might also be able to wash out, at least partly, the unphysical structure, so that a value of $\varepsilon_{\mathrm{ss}} \cong-100 \mathrm{MeV}$ might be closer to the true value.

3) It has been a surprise to realize, when exploring the currents at different energies, that the RPA calculations do not confirm the Goldhaber-Teller model. When the strength is fragmented, the lower peak (to be identified to the experimental one) resembles a Steinwedel Jensen vibration (with a diffuse surface) while the upper peak shows vorticity. The Goldhaber-Teller flow corresponds to a minimum in the strength. When a single peak is obtained, the flow lines still resembles the Steinwedel Jensen model in the interior and they tend to cross 

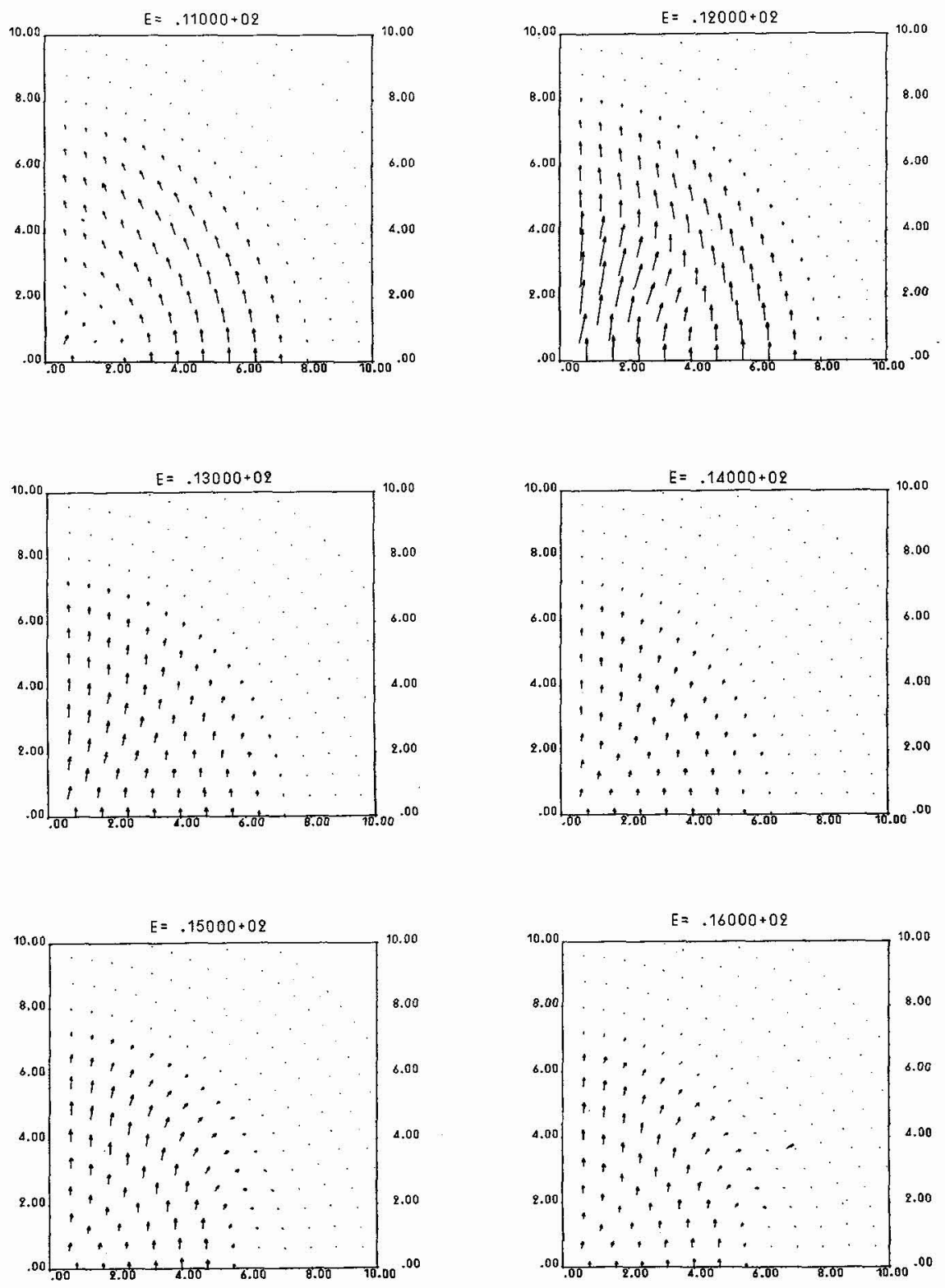

Fig. 12 - Plot of the currents for different energies. one fourth of the nucleus only is considered. The vertical axis is the z-axis. 
the surface in the z-direction. However there is no reason to interpret the resulting flow as a linear superposition of SJ and GT models, as in ref./27/. The method presented in ref./15/ should help understanding this point, when extended to the case of finite nuclei.

Finally let us indicate that the extension of such calculations to finite temperatures is straightforward. Instead of dealing with the energy of the system, one deals with the free energy H-TS and the same formalism then applies (see ref. $/ 37 /)$. However it is not clear to us up to now whether one should consider vibrations of an excited nucleus as being constant-temperature or constantentropy processes. Works in this direction are in progress $/ 38 /$.

The present work results from an earnest collaboration with 0 . Bohigas and H.Krivine. K. Ando, G. Eckart, N. Van Giaj and S. Stringari are acknowledged for useful discussions.

\section{$\checkmark$ - REFERENCES}

11/ BERTSCH G.F., Ann. Phys. 86 (1974) 138

BERTSCH G.F., Nuci. Phys. A2 29 (1975) 253

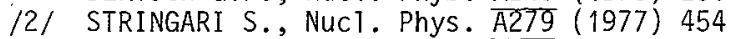

13/ SAGAWA H. and HOLZWARTH G., Progr. Theor. Phys. 59 (1978) 1213

14/ HOLZWARTH G.and ECKART G., Z. Phys. A284 (1978) 291

HOLZWARTH G. and ECKART G., NuCl. Phys. A325 (1979) 1

15/ BLAIZOT J.P., Phys. Rep. 64 (1980) 171

16/ BLAIZOT J.P. and GRAMMATICOS B., Nucl. Phys. A355 (1981) 115

$17 /$ JENNINGS B.K. and JACKSON A.D., Nucl. Phys. A342 (1980) 23

JENNINGS B.K. and JACKSON A.D., Phys. Rep. 66 (1980) 141

18/ TREINER J., KRIVINE H., BOHIGAS 0. and MARTORELL J., NuCl. Phys. A371(1981)253

19/ KRIVINE H., TREINER J. and BOHIGAS 0., Nucl. Phys. A336 (1980) 155

$110 /$ KRIVINE H., SCHMIT C. and TREINER J., Phys. Lett. $1 \overline{12 \mathrm{~B}}$ (1982) 281

111 LIPPARINI E. and STRINGARI S., Phys. Lett. 112B (1982) 421

112/ MEYER J., QUENTIN P. and JENNINGS B.K., NucT. Phys. A385 (1982) 269

$113 /$ STRINGARI S. and LIPPARINI E., Phys. Lett. 117B (1982) 141

144/ HOLZWARTH G. Kyoto summer schoo1,Sept 82, Sup1. Progr.Theor. Hys.n\%4-75(1983)and refs . there in

115/ STRINGARI S., Trento preprint, Sept. 82

/16; ANDO K. and NISHIZAKI S., Progr. Theor. Phys. 68 (1982) 1196

$117 /$ GIANNONI M.J., VAUTHERIN D., VENERONI M. and BRINK D.M., Phys. Lett. 63B( 1976) 8

$118 /$ STRINGARI S., Nucl. Phys. A325 (1979) 199

119/ DA PROVIDENCIA J.P., Ph. D. Thesis, University of Coimbre, 1983, unpublished DA PROVIDENCIA J.P. and HOLZWARTH G., Preprint 1982

$120 /$ SERR F.E., BERTSCH G.F. and BORYSOWICZ J., Phys. Lett. 92B (1980) 241

121/ ECKART G., HOLZWARTH G. and SCHWESINGER B., Phys. Lett. 94B (1980) 453

ECKART G., HOLZWARTH G. and DA PROVIDENCIA J.P., NuCl. Phys. A364 (1981) 1

122/ BRACK M. and STOCKER W., Nuc1. Phys. A406 (1983) 413

123/ DECOWSKI P., MORSCH H.P. and SÜKOSD C., Contribution to this Conference.

124/ BOHIGAS 0., LANE A.M. and MARTORELL J., Phys. Rep. 51 (1979) 267

125/ LEBRUN D., Thèse d'Etat, ISN-GrenobTe, 1981

126/ MYERS W.D., Droplet ModeT of Atomic Nuclej, Plenum Press New York (1977)

/27/ MYERS W.D., SWIATECKI W.J., KODAMA T., EL JAICK L. and HILF E., Phys. Rev. C15 (1977) 2032

128/ LASZEWSKI R.M. and AXEL P., Phys. Rev. C19 (1979) 342

129/ BOHIGAS 0., VAN GIAI N. and VAUTHERIN D., Phys. Lett. 102B (1981) 105

130/ BARTEL J., QUENTIN P., BRACK M., GUET C. and HAKANSON H.B., NUCl. Phys. A386 (1982) 79

131/ BCHIGAS 0. Kyoto surmer schol, Sept.82, Supl.Prog.Theor. Phys. $n^{\circ} 74-75$ (1983)

/32/ BERTSCH G.F. and TSAI S.F., Phys. Rep. 18 (1975) 125

LIU K.F. and BROWN G.E., Nuc7. Phys. A265 (1976) 385

BLAIZOT J.P. , Thèse d'Etat, Université de Paris, 1977

AUERBACH N. and KLEIN A., NuCl. Phys. A395 (1983) 77

VAN GIAI N., private communication 
/33/ BORTIGNON P.F. and BROGLIA R.A., Nuct. Phys. A371 (1981) 405 WAMBACH J., These proceedings

134/ KOCH H., ECKART G., SCHWESINGER B. and HOLZWARTH G., NuCl. Phys. A373 (1982) 173

135/ VAUTHERIN D., private communication

/36/ KRIVINE H. and TREINER J., Phys. Lett. 124B (1983) 127

137/ MEYER J., QUENTIN P. and BRACK M., 7e Session d'Etudes Biennale de Physique Nucléaire, Aussois, France (1983)

138/ BARRANCO M., KRIVINE H., MARCOS S. and TREINER J., in preparation. 